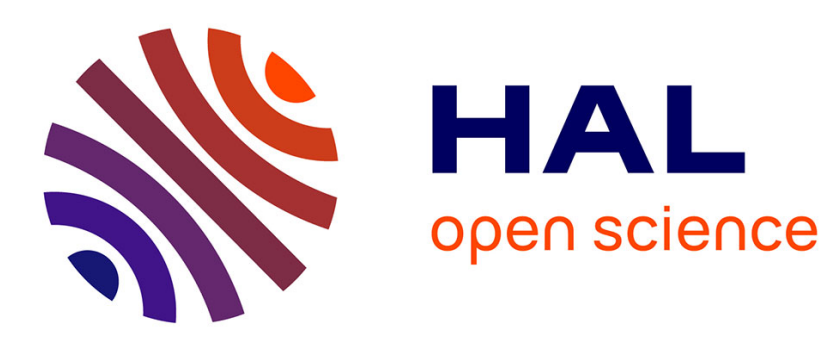

\title{
Recomposition d'un faisceau laser gaussien en une répartition homogène de grande densité d'énergie
}

\author{
J.C. Li, J. Merlin, J. Perez
}

\section{To cite this version:}

J.C. Li, J. Merlin, J. Perez. Recomposition d'un faisceau laser gaussien en une répartition homogène de grande densité d'énergie. Revue de Physique Appliquée, 1987, 22 (3), pp.201-204. 10.1051/rphysap:01987002203020100 . jpa-00245531

HAL Id: jpa-00245531

https://hal.science/jpa-00245531

Submitted on 1 Jan 1987

HAL is a multi-disciplinary open access archive for the deposit and dissemination of scientific research documents, whether they are published or not. The documents may come from teaching and research institutions in France or abroad, or from public or private research centers.
L'archive ouverte pluridisciplinaire HAL, est destinée au dépôt et à la diffusion de documents scientifiques de niveau recherche, publiés ou non, émanant des établissements d'enseignement et de recherche français ou étrangers, des laboratoires publics ou privés. 


\title{
Recomposition d'un faisceau laser gaussien en une répartition homogène de grande densité d'énergie
}

\author{
J. C. Li (*), J. Merlin et J. Perez \\ INSA de Lyon, GEMPPM, U.A. 341, CALFETMAT, Bât. 403, 69621 Villeurbanne Cedex, France
}

(Reçu le 26 mai 1986, accepté le 9 décembre 1986)

\begin{abstract}
Résumé. - Dans une publication précédente l'étude théorique comparative de dispositifs permettant de transformer un faisceau laser $\mathrm{TEM}_{00}$ en une répartition uniforme a montré les qualités du dispositif à miroir désigné « $L_{1}$ ». Nous montrons que les performances de ce dispositif peuvent être accrues par adjonction d'un système dioptrique permettant d'obtenir des densités d'énergie plus élevées sans altération notable de la répartition d'énergie.

Abstract. - In previous publication the theoritical study of several devices able to transform an $\mathrm{TEM}_{00}$ laser beam in an uniform energy distribution has shown the quality of a mirors device called « $\mathrm{L}_{1}$ ». Here it shown that the performances of this device can be increased by the adjunction of lenz allowing to obtain higher energy density without high alteration of energy distribution.
\end{abstract}

\section{Introduction.}

Une étude précédente [1] portant sur la comparaison de différents dispositifs permettant la transformation d'un faisceau laser, ayant une répartition d'énergie gaussienne, en une répartition carrée homogène, a montré qu'un dispositif (désigné « $\mathrm{L}_{1}$ ) composé d'un miroir prismatique à 4 faces et de 4 miroirs sphériques devait permettre, par superposition d'images de 4 quarts du faisceau, d'obtenir un résultat de bonne qualité.

Toutefois, la dimension du faisceau ainsi reconstitué ne peut être que du même ordre de grandeur que celle du faisceau incident, par conséquent, la densité maximale de puissance atteinte sera relativement faible (de l'ordre de $10^{3} \mathrm{~W} / \mathrm{cm}^{2}$ dans le cas d'une source de $1 \mathrm{~kW}$ avec un faisceau de l'ordre de $10 \mathrm{~mm}$ de diamètre, caractéristiques habituelles pour des sources $\mathrm{CO}_{2}$ continu à faisceau $\mathrm{TEM}_{00}$ ). Cela est insuffisant pour nombre d'applications, même du type trempe superficielle d'aciers [2] pour lesquelles des densités de $10^{4} \mathrm{~W} / \mathrm{cm}^{2}$ sont habituellement requises. En plaçant une lentille sur le parcours du faisceau incident il est possible de diminuer le grandissement global du système $(G)$ et par suite, d'obtenir une tache de dimension plus réduite ; reste à préciser si la qualité de la répartition énergétique ne se trouve pas trop altérée par l'introduction de cet élément d'optique supplémentaire. Le présent travail

(*) Adresse permanente: Institut de Technologie de Kun 'Ming, Yunnam, République Populaire' de Chine. a pour objet de réaliser cette analyse d'un point de vue théorique.

\section{Calcul de la répartition d'énergie.}

Le principe de calcul reste analogue à celui présenté dans le paragraphe 3.1 de la publication précédente [1].

La figure 1 représente le chemin optique équivalent du dispositif lentille $+\ll \mathrm{L}_{1} »$. Les plans objets par rapport aux miroirs sphériques sont toujours définis comme étant les plans passant par le sommet du prisme et perpendiculaires à la direction des 4 sous-faisceaux réfléchis. Après traversée de la lentille le faisceau incident est considéré comme convergent vers le foyer de la lentille (en fait la surface d'onde correspondant à un faisceau $\mathrm{TEM}_{00}$ est théoriquement un ellipsoïde de révolution et le centre du plan de convergence du faisceau peut être considéré comme le foyer).

Supposons que l'amplitude au centre du faisceau laser incident ait un module unité avant la traversée de la lentille, le champ dans le plan-objet équivalent peut s'écrire directement (voir Fig. 1) :

$$
\begin{aligned}
U_{0}\left(X_{0}, Y_{0}\right)=1 / M_{0} \times & \exp \left[\left(-j k / 2 F_{0}\right)\left(X_{0}^{2}+Y_{0}^{2}\right)\right] \times \\
& \times \exp \left[-\left(X_{0}^{2}+Y_{0}^{2}\right) /\left(M_{0} W\right)^{2}\right]
\end{aligned}
$$

avec

$$
\begin{aligned}
& 0 \leqslant X_{0} \leqslant 2 M_{0} W \\
& 0 \leqslant Y_{0} \leqslant 2 M_{0} W .
\end{aligned}
$$

$k=2 \pi / \lambda, \lambda$ étant la longueur d'onde du faisceau incident et $W$ le rayon de ce faisceau; 
$M_{0}=F_{0} / f_{0}$, où $F_{0}$ est la distance séparant le sommet du miroir prismatique du foyer de la lentille et $f_{0}$ la distance focale de la lentille.

Si $M$ est le grandissement transversal de l'image donnée par les miroirs sphériques, la répartition énergétique prévue par l'optique géométrique au niveau du champ image sera :

$$
\begin{aligned}
1 / M \cdot U_{0}\left(-X_{\mathrm{i}} / M,\right. & \left.-Y_{\mathrm{i}} / M\right)=1 / M M_{0} \times \\
& \times \exp \left[-j\left(k / 2 F_{0} M^{2}\right)\left(X_{\mathrm{i}}^{2}+Y_{\mathrm{i}}^{2}\right)\right] \\
& \times \exp \left[-\left(X_{\mathrm{i}}^{2}+Y_{\mathrm{i}}^{2}\right) /\left(M M_{0} W\right)^{2}\right]
\end{aligned}
$$

avec

$$
\begin{aligned}
& -2 M M_{0} W \leqslant X_{\mathrm{i}} \leqslant 0 \\
& -2 M M_{0} W \leqslant Y_{\mathrm{i}} \leqslant 0 .
\end{aligned}
$$

D'après l'optique de Fourier, la fonction image $U_{\mathrm{i}}\left(X_{\mathrm{i}}, Y_{\mathrm{i}}\right)$, dans le plan image, est égale au produit de convolution de la réponse impulsionnelle du système par la fonction image prévue par l'optique géométrique.

$$
\begin{aligned}
U_{\mathrm{i}}\left(X_{\mathrm{i}}, Y_{\mathrm{i}}\right)=\iint_{-2 M M_{0} W}^{0} 1 / M M_{0} \exp \left[-j\left(k / 2 F_{0} M^{2}\right)\left(X^{2}+Y^{2}\right)\right] \exp \left[-\left(X^{2}+Y^{2}\right) /\left(M M_{0} W\right)^{2}\right] \times \\
\quad \times \sin \left[(\pi / T)\left(X-X_{\mathrm{i}}\right)\right] / \pi\left(X-X_{\mathrm{i}}\right) \cdot \sin \left[(\pi / T)\left(Y-Y_{\mathrm{i}}\right)\right] / \pi\left(Y-Y_{\mathrm{i}}\right) \cdot \mathrm{d} X \mathrm{~d} Y
\end{aligned}
$$

où $T=\lambda d_{\mathrm{i}} / 2 a$ avec $d_{\mathrm{i}}$ distance image.

Tant que l'inclinaison $\varphi$ du plan image par rapport au plan de superposition est faible, et l'on essaiera de rester dans cette situation, l'effet de projection pourra être négligé. En faisant une translation du système d'axe on obtient alors le champ image dans

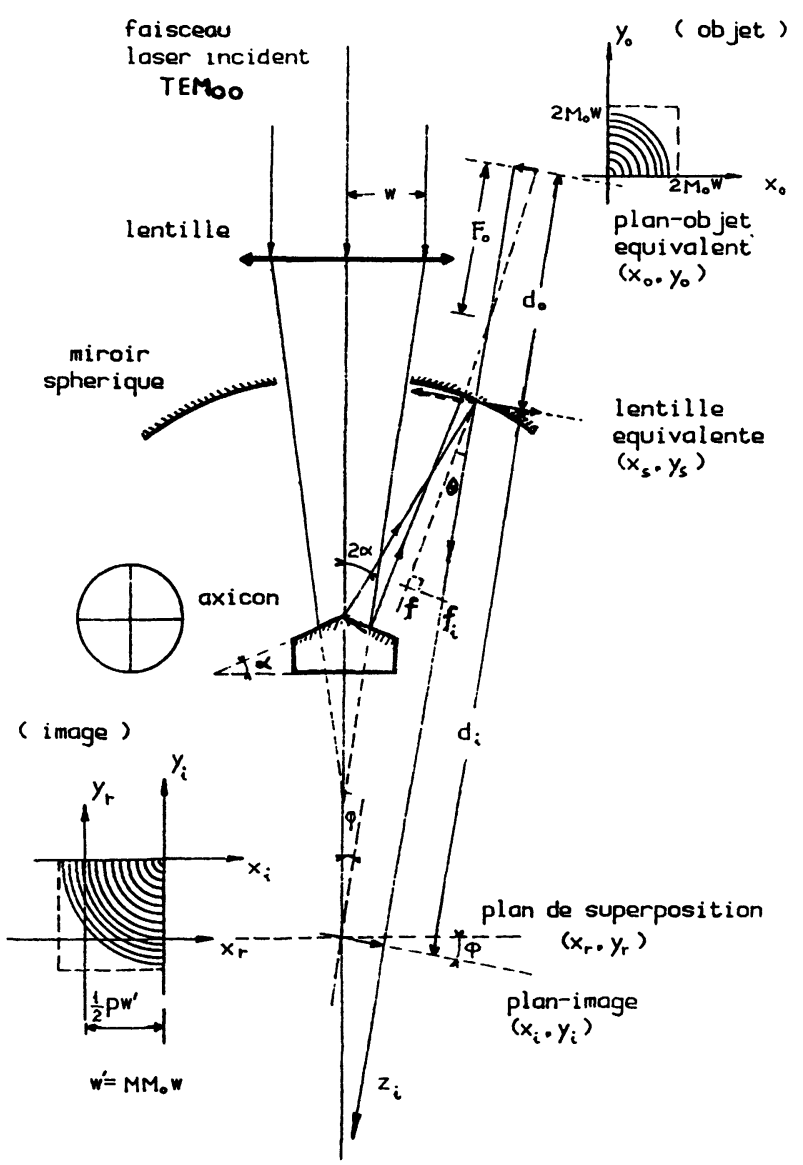

Fig. 1. - Représentation du chemin optique équivalent pour le dispositif étudié.

[Equivalent optic paths for the studied device.] le plan de superposition perpendiculaire à l'axe du système.

$$
U\left(X_{\mathrm{r}}, Y_{\mathrm{r}}\right)=U_{\mathrm{i}}\left(X_{\mathrm{r}}-p W^{\prime} / 2, Y_{\mathrm{r}}-p W^{\prime} / 2\right)
$$

où $p W^{\prime}$ représente la dimension du côté de la tache formée avec $W^{\prime}=M M_{0} W$. Le coefficient de proportionnalité $p$ définit la position relative des 4 images des sous-faisceaux et il a été montré [3] que la valeur $p=1,1$ était optimale pour obtenir une superposition des 4 sous-faisceaux conduisant à une tache bien carrée avec une répartition homogène.

La répartition d'énergie après superposition des sous-faisceaux sera donnée par:

$$
\begin{aligned}
I\left(X_{\mathrm{r}}, Y_{\mathrm{r}}\right)=U\left(X_{\mathrm{r}},\right. & \left.Y_{\mathrm{r}}\right) U^{*}\left(X_{\mathrm{r}}, Y_{\mathrm{r}}\right)+ \\
& +U\left(-X_{\mathrm{r}}, Y_{\mathrm{r}}\right) U^{*}\left(-X_{\mathrm{r}}, Y_{\mathrm{r}}\right) \\
& +U\left(-X_{\mathrm{r}},-Y_{\mathrm{r}}\right) U^{*}\left(-X_{\mathrm{r}},-Y_{\mathrm{r}}\right) \\
& +U\left(X_{\mathrm{r}},-Y_{r}\right) U^{*}\left(X_{\mathrm{r}},-Y_{\mathrm{r}}\right) .
\end{aligned}
$$

\section{Résultats et conclusion.}

Le calcul numérique de la répartition d'énergie a été effectué avec des paramètres analogues à ceux pris dans l'étude précédente de « $L_{1}$ » [1] pour différentes valeurs du grandissement global $G=M \cdot M_{0}$ à savoir : 1,5-1 et 0,5 (voir Fig. 2), une analyse plus détaillée étant effectuée dans la situation la plus intéressante $G=0,5$ (voir Fig. 3).

On doit remarquer que $G=1,5$ équivaut à la situation de la publication précédente en l'absence de lentille avec $M=1,5$. Les deux autres cas étudiés peuvent, quant à eux, correspondre à des situations où $M=1,5$ et $M_{0}=2 / 3$ ou $1 / 3$.

Les niveaux moyens $H$ des densités d'énergie maximales observés varient bien comme $\left(1 / M_{0}\right)^{2}$, le gain d'un ordre de grandeur en densité d'énergie impose donc une valeur de $M_{0}$ de l'ordre de $1 / 3$. L'adjonction d'une lentille supplémentaire au dispo- 


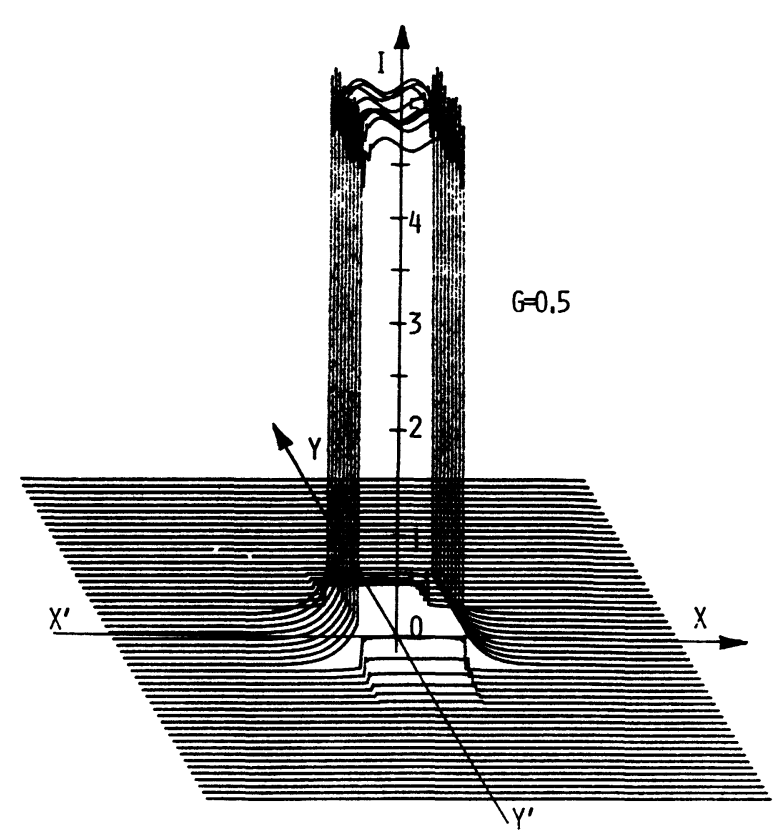

Fig. 2. - Coupe axiale de la répartition d'énergie dans le plan de superposition pour différentes valeurs du grandissement.

[Schematic axial section of energy distribution in the recombination plane for different values of optical magnification $G$.]

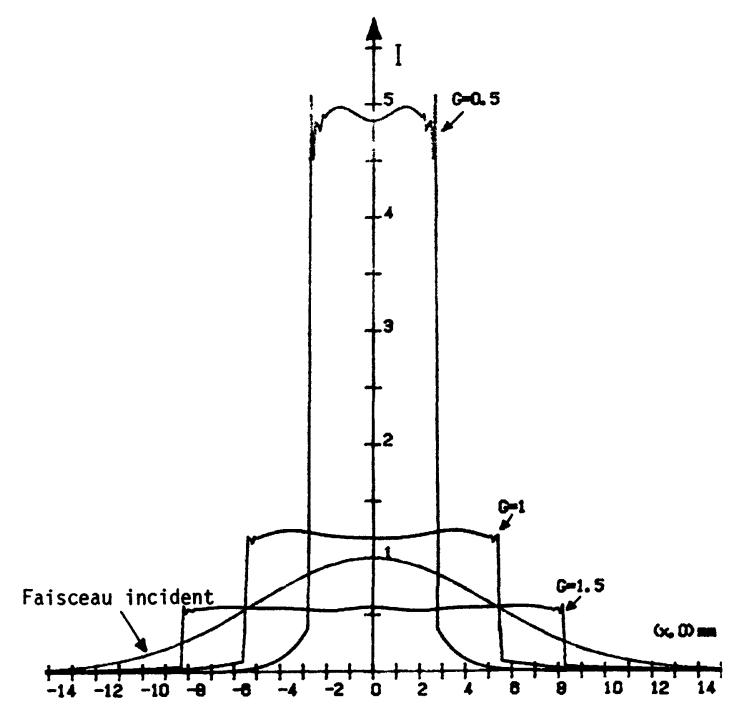

Fig. 3. - Répartition d'énergie dans le plan de superposition pour un grandissement de 0,5 .

[Intensity distribution in the recombination plane for an optical magnification $G=0.5$.]

sitif « $\mathrm{L}_{1}$ » permet d'atteindre des niveaux de densité d'énergie comparables à ceux que l'on est susceptible d'obtenir avec le dispositif « $L_{2}$ » étudié précédemment [1] et qui utilisait une simple superposition de faisceaux sans formation d'image.

Cette solution étant nettement plus complexe que le dispositif $\ll L_{2}$ » reste à savoir si au niveau des fluctuations dans la répartition d'énergie au sein de la tache reconstituée on conserve les qualités qui présentaient le dispositif « $\mathrm{L}_{1}$ » par rapport à $\ll \mathrm{L}_{2} »$.

Nous n'avons pas effectué une étude exhaustive dans ce domaine, cependant une analyse numérique correspondant à la situation la plus intéressante (à savoir $G=0,5$ ) permet de mettre en évidence les points suivants :

- l'amplitude maximale $h / H$ des fluctuations de répartition d'énergie est d'environ $5 \%$. Il n' y a donc pas de détérioration par rapport au dispositif primitif sans lentilles et les fluctuations relatives de température $\Delta T / T$ susceptibles d'être induites resteront de 4 à 5 fois plus faibles que celles résultant de l'utilisation du dispositif « $\mathrm{L}_{2} »$;

- la périodicité $\Delta_{K}$ des fluctuations est de l'ordre du dixième de la largeur de la tache et les fluctuations s'étendent à l'ensemble de la tache. On observe donc une nette dégradation vis-à-vis du dispositif « $\mathrm{L}_{1}$ » et on a un comportement qui se rapproche de celui observé pour « $\mathrm{L}_{2}$ ";

- en ce qui concerne la largeur $\Delta$ sur laquelle s'effectue la montée en énergie on observe également un comportement proche de celui de $« \mathrm{~L}_{2}$ ».

En conclusion l'introduction d'une lentille dans le dispositif « $L_{1}$ » fait incontestablement perdre une certaine partie des qualités de ce dispositif vis-à-vis de la répartition d'énergie au niveau de la tache reconstituée, mais apporte cependant une amélioration nette vis-à-vis de dispositif plus simple du type « $\mathrm{L}_{2}$ ». Afin de faciliter cette comparaison nous avons ainsi rassemblé sur la figure 4 les répartitions

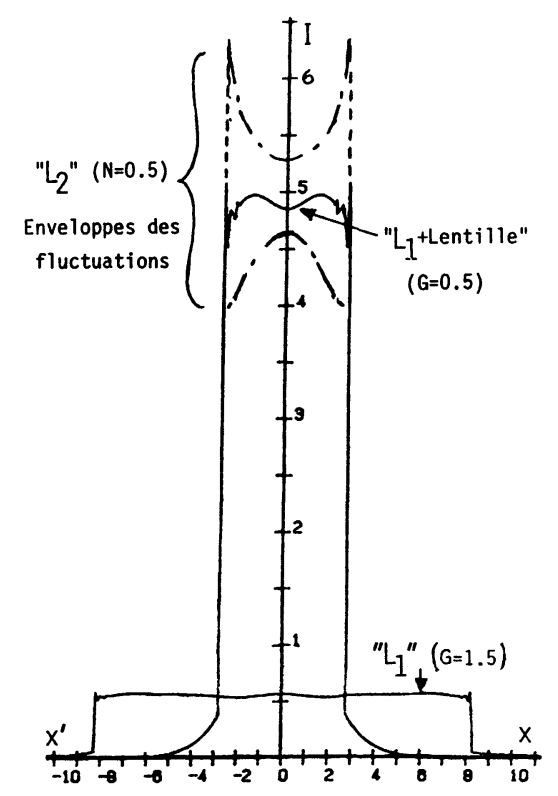

Fig. 4. - Comparaison des répartitions d'énergie obtenues avec les différents dispositifs proposés.

[Comparison between the energies distributions obtained with the different devices.] 
d'énergie calculées pour le dispositif « $L_{1}$ » initial, le dispositif « $\mathrm{L}_{1}$ » + lentille : $G=0,5$ (qui permet de multiplier d'un facteur $\simeq 10$ la densité moyenne d'énergie), ainsi que les courbes enveloppes des limites de fluctuations de répartition d'énergie correspondant à un dispositif « $\mathrm{L}_{2}$ » de grandissement transversal $N=0,5$ donnant le même niveau moyen de densité d'énergie.

Evidemment les aberrations introduites par la lentille n'ont pas été ici prises en compte et une étude pratique d'un tel dispositif devra être effectuée pour préciser les limitations réelles de ce dispositif; cette approche théorique permet cependant avec une source $\mathrm{CO}_{2}$ continue, gaussienne, de $4 \mathrm{~kW}$ et de
$14 \mathrm{~mm}$ de diamètre (caractéristiques approximatives de la source CILAS CI 4000 à $5 \mathrm{~m}$ de la sortie de la cavité), d'envisager d'obtenir des densités homogènes de l'ordre de $40 \pm 1 \mathrm{~kW} / \mathrm{cm}^{2}$ sur une tache d'un peu plus de $3 \mathrm{~mm}$ de côté (c'est-à-dire de traiter une surface appréciable à, par exemple, $1000 \pm 25^{\circ} \mathrm{C}$ ) ; un tel résultat, s'il est atteint, correspond tout à fait aux exigences des traitements thermiques superficiels. En fait, le plus gros problème réside certainement dans la tenue du prisme quadrifacial aux hautes densités de puissance (quelque $10^{4} \mathrm{~W} / \mathrm{cm}^{2}$ ) et là encore seule une étude pratique, d'ailleurs en cours dans notre laboratoire, permettra d'apporter une réponse.

\section{Bibliographie}

[1] Li, J. C., Merlin, J., Perez, J., Revue Phys. Appl. 21 (1986) 425-433.

[2] Source Book on applications of the Laser in Metalworking (A.S.M.) 1981.

[3] Girardeau-Montaut, J. C., Li, J. C., GirardeauMontaut, C., Optics Commun., janvier 1986. 